\title{
BMJ Open Is mortality-to-incidence ratio associated with health disparity in pancreatic cancer? A cross-sectional database analysis of 57 countries
}

\author{
Hsiang-Lin Lee, ${ }^{1,2,3}$ Cheng-Ming Peng, ${ }^{1,2,3}$ Cheng-Yu Huang, ${ }^{4}$ Shin-Yi Wu, ${ }^{5}$ \\ Ming-Chang Tsai, ${ }^{1,2,6}$ Chi-Chih Wang, ${ }^{1,2,6}$ Sung-Lang Chen, ${ }^{1,2,7}$ Chun-Che Lin, ${ }^{1,2,6}$ \\ Chien-Ning Huang, ${ }^{1,2,8}$ Wen-Wei Sung ${ }^{1,2,7}$
}

To cite: Lee H-L, Peng C-M, Huang C-Y, et al. Is mortalityto-incidence ratio associated with health disparity in pancreatic cancer? A crosssectional database analysis of 57 countries. BMJ Open 2018;8:e020618. doi:10.1136/ bmjopen-2017-020618

- Prepublication history and additional material for this paper are available online. To view these files, please visit the journal online (http://dx.doi. org/10.1136/bmjopen-2017020618).

H-LL and C-MP contributed equally.

Received 14 November 2017 Revised 17 May 2018 Accepted 6 June 2018

Check for updates

For numbered affiliations see end of article.

Correspondence to

Dr Wen-Wei Sung;

flutewayne@gmail.com

\section{ABSTRACT}

Objective The colorectal cancer mortality-to-incidence ratio (MIR) can reflect healthcare disparities. However, a similar association has not yet been established between the MIR of pancreatic cancer and healthcare disparities.

Methods In this study, the incidence and mortality rates of pancreatic cancer were obtained from the GLOBOCAN 2012 database. The WHO rankings and total expenditures on health/gross domestic product (e/GDP) were obtained from a public database. Linear regression was performed to determine correlations between the variables.

Results 57 countries met the inclusion criteria according to the data quality. Developed regions (Europe and the Americas) had high pancreatic cancer incidence and mortality rates. The MIRs were over 0.90 in all regions. No significant correlation was found between MIRs and the WHO rankings, e/GDP or per capita total expenditure on health for analysis in the 57 countries, indicating no association between MIRs and cancer care disparities for pancreatic cancer.

Conclusions The MIR variations for pancreatic cancer do not correlate with healthcare disparities among countries. Further investigation is necessary to confirm this observation with secondary analysis of databases.

\section{INTRODUCTION}

Pancreatic cancer is a highly lethal disease, and most cases are usually detected in the advanced stages, after metastasis. ${ }^{1}$ The incidence rates are currently increasing in the $\mathrm{USA}^{2}{ }^{2}$ as well as in European countries, highlighting a rising trend worldwide for this disease that might be associated with improved diagnosis methods. ${ }^{34}$ However, patients with advanced pancreatic cancer have limited treatment options, and fewer than $20 \%$ are candidates for surgery. ${ }^{56}$ Consequently, the prognosis is poor for patients diagnosed with pancreatic cancer.

Many cancers differ in their incidence and mortality rates in specific countries, regions and continents across the globe, which raises
Strengths and limitations of this study

- This is a large observational study with secondary analysis of databases using a global database containing 184 countries.

- Only countries with relatively good data quality are included in further analysis.

- This study did not explore the clinical diversity of patients.

- This is not a cohort study; therefore, the mortality and incident cases might not represent the same patients.

No information about survival time and survival rate was provided in this analysis.

the possibility that these differences could reflect regional healthcare disparities. ${ }^{7-10}$ The aim of the present study was to use the mortality-to-incidence ratio (MIR) as a parameter for evaluating pancreatic cancer in different countries. The MIR is a measurement that compares the relationship between mortality and incidence, and its value can serve as a proxy for 1 -survival. ${ }^{11} 12$ Achieving low MIR values depends on adequate cancer screenings, early diagnosis and effective treatments by the healthcare system. ${ }^{13}$ For example, Sunkara and Hebert, in their study on colorectal cancer, described the MIR as a useful indicator for cancer screening and care in patients with colorectal cancer, as they found a positive correlation between the MIR values and the healthcare system rankings for different countries. ${ }^{10}$

Pancreatic cancer, unlike colorectal cancer, is difficult to detect and lacks routine screening policies. ${ }^{151415}$ No evidence yet supports an association between cancer health disparities and the MIR of patients with this cancer. The objective of our study was therefore to evaluate the relationships 
between the incidence and mortality rates of pancreatic cancer and healthcare parameters, such as total expenditures on health/gross domestic product (e/GDP) and WHO rankings.

\section{MATERIALS AND METHODS \\ Data acquisition}

Cancer epidemiological data were obtained from the GLOBOCAN 2012 database maintained by the International Agency for Research on Cancer (https://www.iarc. $\mathrm{fr} /$ ). The WHO ranking for these countries was obtained from the World's Health Systems, maintained by WHO. The e/GDP, per capita total expenditure on health and life expectancies for 2012 were obtained from the World Health Statistics 2015, which is an annual compilation of health-related data for its 194 member states. In total, 184 countries were screened in the GLOBOCAN 2012 database. Among these, 25 countries were excluded since no matching data existed in the WHO database. We also excluded 102 countries according to the availability level rankings of the mortality and incidence data in the GLOBOCAN 2012 database; these excluded countries had availability level rankings of E-G for incidence or 4-6 for mortality. In total, 57 countries were ultimately analysed. The crude rate was defined as rate every 100000 persons. The MIR, in the present study, was defined as the ratio of the crude rate of mortalities and the crude rate of incidences. ${ }^{10}$

\section{Patient and public involvement}

This study analysed GLOBOCAN 2012 database. Therefore, we did not inform or disseminate the patients about the research question, outcome measures and results. Patients did not involve in the study, including design, recruitment and conduct of the study. There was no patient adviser for contributorship statement.

\section{Statistical analyses}

Associations between the MIRs and variants among countries were analysed by linear regression as described previously. ${ }^{7} \mathrm{R}^{2}$ changes and analysis of variance were analysed using SPSS statistical software version 15.0 (SPSS, Chicago, Illinois, USA). P values $<0.05$ of two-sided tests were considered statistically significant. Scatter plots were generated via Microsoft Excel 2010.

\section{RESULTS}

Rates of incidence/mortality and MIR of pancreatic cancer according to regions

The case numbers and rates of incidence and mortality for pancreatic cancer in different regions of the world are summarised in table 1 . These regions were determined by

Table 1 Summary of the case numbers, rates and mortality-to-incidence ratio (MIR) of the incidence and mortality according to regions in pancreatic cancer

\begin{tabular}{|c|c|c|c|c|c|c|c|}
\hline \multirow[b]{2}{*}{ Region } & \multicolumn{2}{|l|}{ Number } & \multicolumn{2}{|l|}{ Crude rate } & \multicolumn{2}{|c|}{ Age-standardised rate } & \multirow[b]{2}{*}{ MIR $^{*}$} \\
\hline & Incidence & Mortality & Incidence & Mortality & Incidence & Mortality & \\
\hline World & 337872 & 330391 & 4.8 & 4.7 & 4.2 & 4.0 & 0.98 \\
\hline More developed regions & 187465 & 184429 & 15.0 & 14.8 & 7.2 & 6.8 & 0.99 \\
\hline Less developed regions & 150407 & 145962 & 2.6 & 2.5 & 2.8 & 2.7 & 0.96 \\
\hline WHO Africa region & 8324 & 8048 & 0.9 & 0.9 & 1.8 & 1.7 & 1.00 \\
\hline WHO Americas region & 75094 & 73751 & 7.9 & 7.7 & 5.9 & 5.6 & 0.98 \\
\hline $\begin{array}{l}\text { WHO East Mediterranean } \\
\text { region }\end{array}$ & 7686 & 7440 & 1.2 & 1.2 & 1.9 & 1.8 & 1.00 \\
\hline WHO Europe region & 110499 & 111029 & 12.2 & 12.3 & 6.5 & 6.4 & 1.01 \\
\hline Africa & 12101 & 11704 & 1.1 & 1.1 & 2.0 & 1.9 & 1.00 \\
\hline Latin America and Caribbean & 27723 & 27935 & 4.6 & 4.6 & 4.4 & 4.4 & 1.00 \\
\hline Northern America & 47371 & 45816 & 13.5 & 13.1 & 7.4 & 6.9 & 0.97 \\
\hline Asia & 143363 & 137251 & 3.4 & 3.2 & 3.2 & 3.0 & 0.94 \\
\hline Europe & 103845 & 104554 & 14.0 & 14.1 & 6.8 & 6.6 & 1.01 \\
\hline Oceania & 3469 & 3131 & 9.2 & 8.3 & 5.9 & 5.2 & 0.90 \\
\hline
\end{tabular}

${ }^{*}$ The percentage in the ratio of the crude rate of mortalities and the crude rate of incidences. 


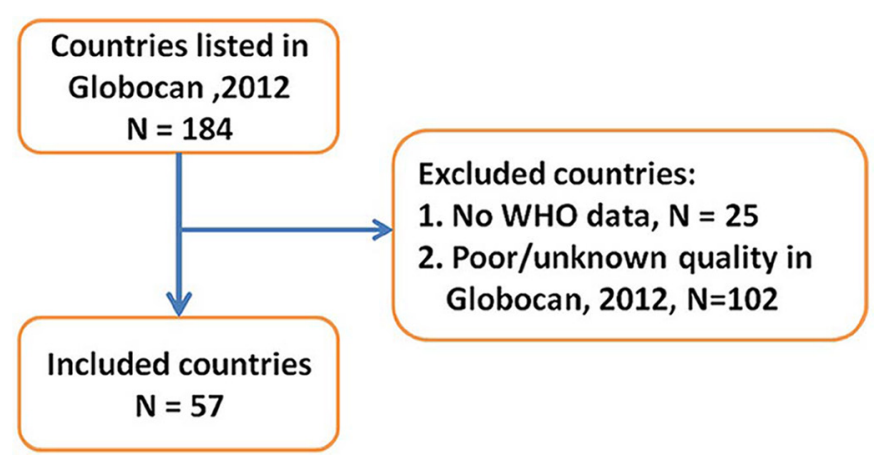

Figure 1 Diagram for data source selection.

three categories: development status, WHO region categories and continent. In this database, the incidence of pancreatic cancer is close in number (337872 cases) to the number of mortalities (330391 deaths). The world, as a whole, has a crude incidence rate of 4.8 and a crude mortality rate of 4.7. The age-standardised rates (ASRs) are 4.2 and 4.0 for incidence and mortality, respectively. The crude rates and ASRs of incidence and mortality are much larger in developed regions than in less developed regions (table 1). For the WHO region categories, the crude rates and ASRs of incidence and mortality are highest in the European region. Africa has the lowest crude rates and ASRs among the continents. The overall MIR is 0.98 , and high MIRs occur in all regions (table 1 ).

\section{Rates of incidence/mortality and MIR of pancreatic cancer according to countries}

In total, 57 countries were included according to the criteria in this study (figure 1). Table 2 summarises the incidence and mortality according to country. The USA has the highest case number among the 57 countries. Four countries have a crude rate of incidence greater than 20: Japan (26.0), Finland (21.3), Germany (20.1) and the Czech Republic (20.0). These countries are also among the five that have crude mortality rates over 18 , with the other one being Slovenia (18.3). The Czech Republic shows the highest ASR in both incidence and mortality (9.7 and 8.7, respectively).

The calculated MIRs are also presented in table 2 . Malta has the lowest MIR among the 57 countries. Eighteen countries have MIRs greater than 1.00, with Sweden having the highest MIR. Eight countries have MIRs below 0.90, including Malta (0.80), Costa Rica (0.84), Bahrain (0.87), Bulgaria (0.85), Denmark (0.86), the Philippines (0.88), Ukraine (0.89) and Australia (0.89).

\section{The associations between WHO ranking, e/GDP, per capita total expenditure on health and life expectancy among countries}

The WHO ranking, e/GDP and life expectancy among the different countries are also listed in table 2. Linear regression confirms that countries with better WHO rankings have a high e/GDP $(\mathrm{p}=0.012$, online supplementary figure S1A). A similar relationship is evident between the WHO ranking and life expectancy $(p<0.001$, online supplementary figure S1B). Countries with better WHO rankings are more likely to have higher e/GDP and a longer life expectancy.

The associations between crude rates and ASRs of incidence and mortality to the WHO ranking and to e/GDP are demonstrated in online supplementary figures S2 and S3. Countries with better WHO rankings are more likely to have higher incidence and mortality crude rates, but the ASR shows no statistical significance (online supplementary figures S2A-S2D). Countries with higher e/ GDP also have higher incidence and mortality crude rates and ASRs (online supplementary figures S3A-S3D). Interestingly, no significant correlations are observed between WHO ranking, e/GDP and MIR (WHO ranking: $\mathrm{R}^{2}=0.001, \mathrm{p}=0.818$, e/GDP: $\mathrm{R}^{2}=0.006, \mathrm{p}=0.553$ ), as shown in figure 2. This is further confirmed via using per capita total expenditure on health for analysis. The list of per capita total expenditure on health of selected countries is shown in online supplementary table 1 . As shown in figure 3, the MIR is not significantly correlated with per capita total expenditure on health.

\section{DISCUSSION}

To our knowledge, this is the first study to investigate the associations between the MIR of pancreatic cancer, the WHO ranking, life expectancy and e/GDP. We found no correlation between MIRs and WHO rankings or between MIRs and e/GDP. These findings differ from those reported previously for colorectal cancer, where a positive correlation was found between the healthcare system rankings and MIRs. ${ }^{10}$ This discrepancy probably reflects the different characteristics and natural courses of these two cancers. Colorectal cancer is easier to screen with faecal immunochemical testing, fecal occult blood tests(FOBTs) or colonoscopy and it is a slowly developing cancer, taking years to develop from polyps. ${ }^{16}$ The screening methods and long duration of cancer development allow early detection of the disease, as reflected by a higher worldwide incidence rate and an early diagnosis. ${ }^{10}$ Pancreatic cancer, by contrast, has no efficient screening methods comparable with those available for colorectal cancer. ${ }^{141517}$ The sole screening method that is accessible to the public consists of the CA-199 biomarker, which, unfortunately, has a poor sensitivity and specificity since many non-malignant diseases also increase its level. ${ }^{17}{ }^{18} \mathrm{CT}$ is also not sufficiently sensitive for detecting early pancreatic cancer. ${ }^{19}$ The lack of screening and early detection strategies, even in highly developed regions, results in advanced cancer stage at diagnosis, with 55\% of the cases being diagnosed at stage IV. ${ }^{20}$ Consistently high MIRs across global regions are therefore inevitable, as approximately $95 \%$ of the cases die within 5 years after diagnosis. ${ }^{20}$

Conversely, we found negative correlations between the WHO ranking and the e/GDP, life expectancy and incidence and mortality crude rates for pancreatic cancer, meaning that the numbers for these categories would 


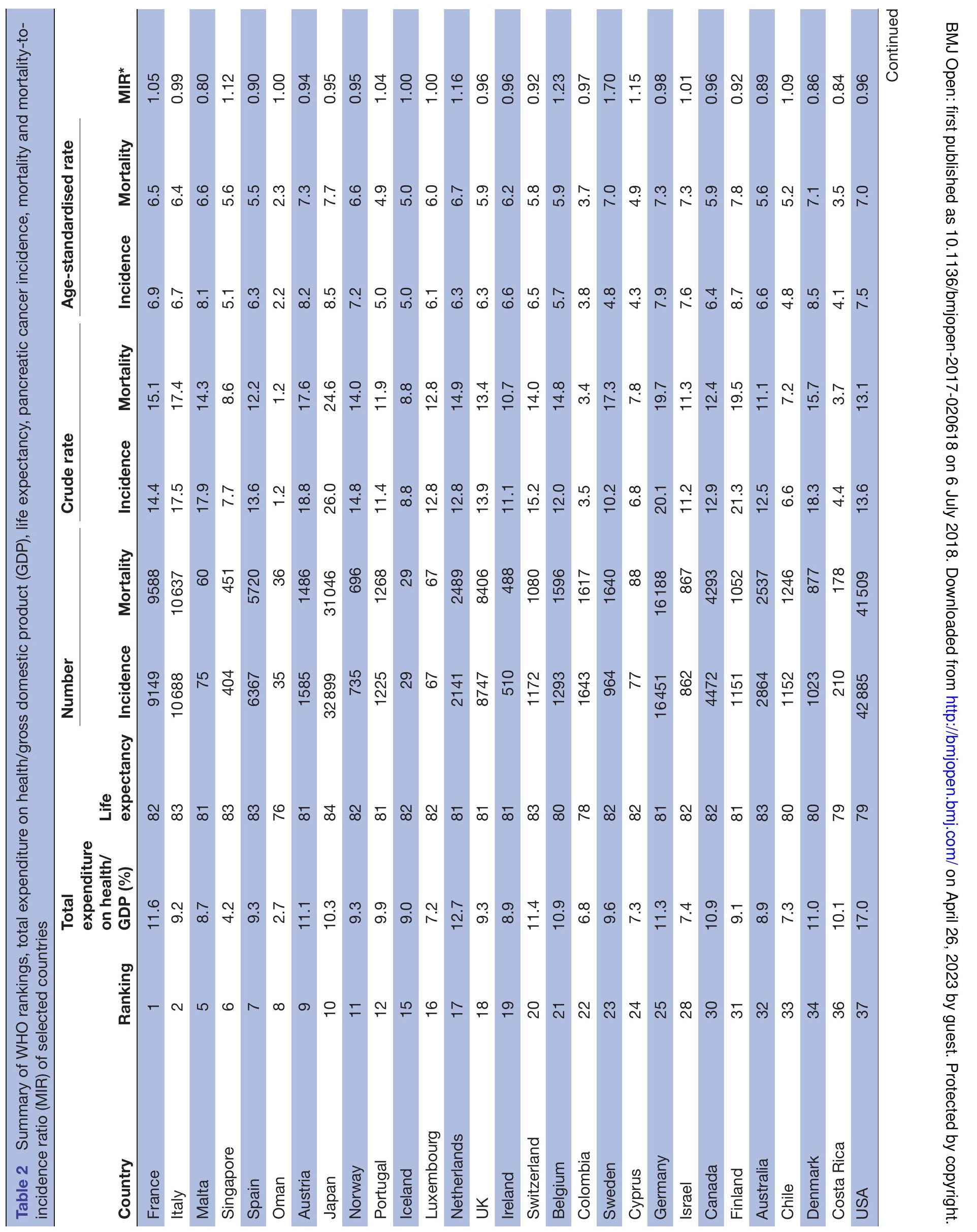


㐍 命

$\frac{\text { ț }}{\frac{ \pm}{\pi}}$

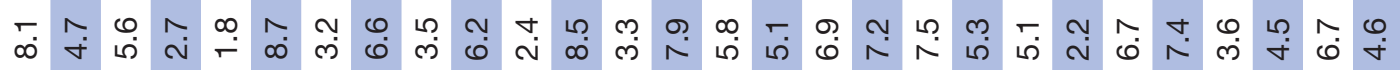

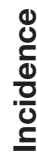

舟

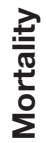

m

$\frac{0}{\pi}$
$\frac{0}{0}$
$\frac{0}{0}$
$\frac{2}{0}$
0

\begin{tabular}{l}
0 \\
$\frac{0}{0}$ \\
$\stackrel{0}{0}$ \\
\hdashline \\
\end{tabular}

我

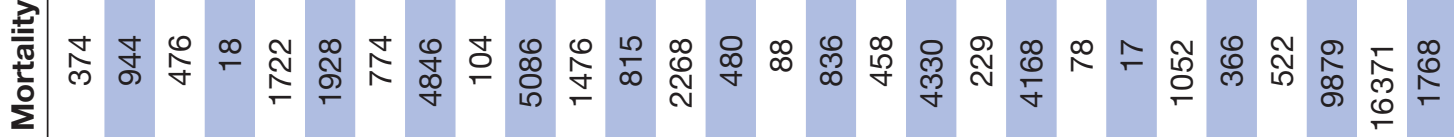

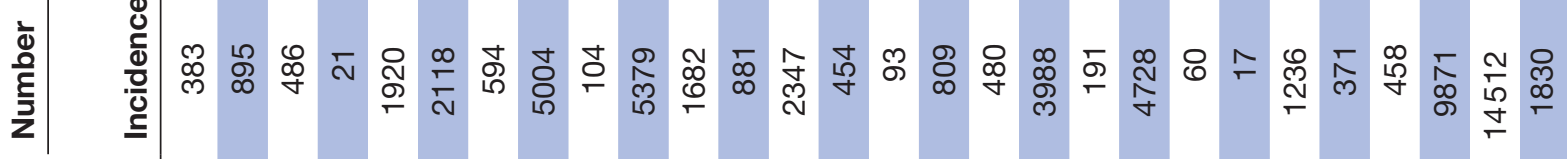

竧

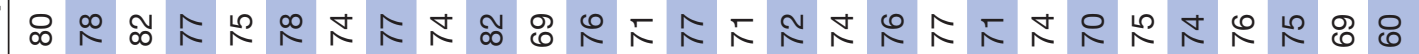

紊 兲

产

离

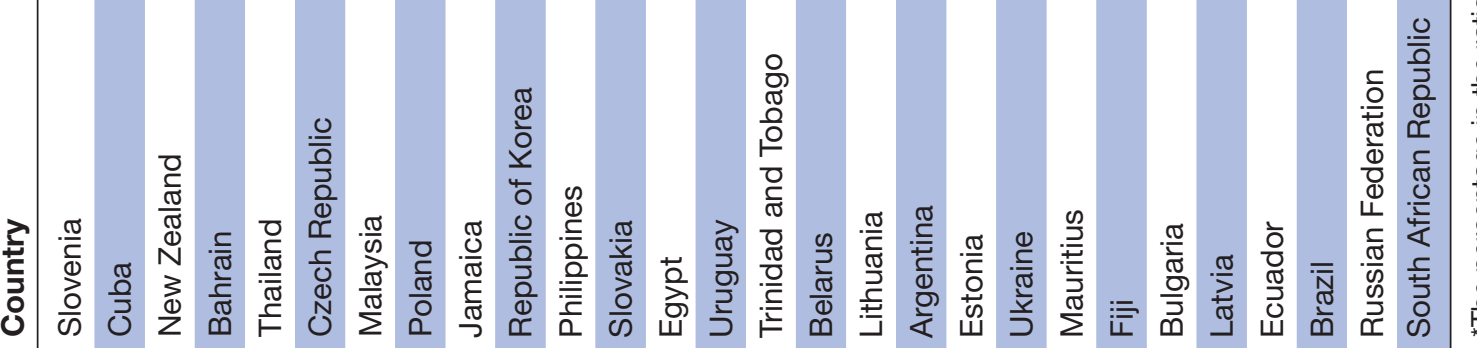


(A)

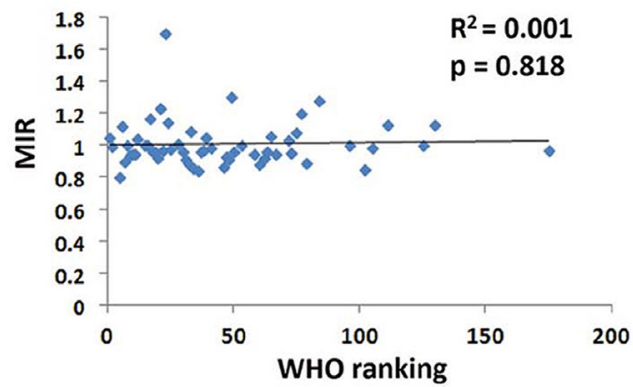

(B)

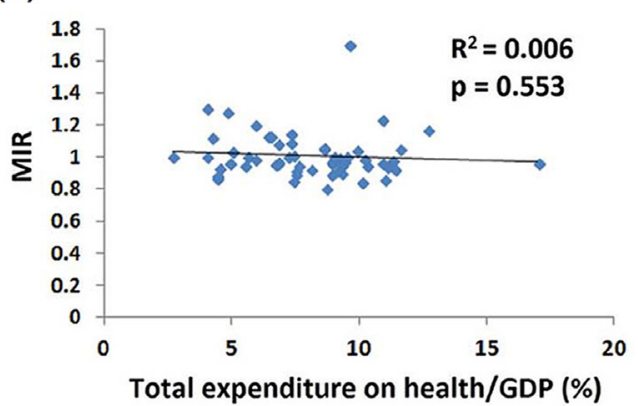

Figure 2 The (A) WHO rankings and (B) total expenditures on health/gross domestic product (GDP) were not associated with the mortality-to-incidence ratio (MIR) of pancreatic cancer.

increase as the WHO rankings improved. We also found positive correlations between the crude rates of mortality and incidence and the e/GDP. ${ }^{72122}$ One possible explanation for this latter finding is that this disease usually strikes the elderly, with most cases diagnosed at ages $60-80 .{ }^{3}$ Our finding of a negative correlation between the WHO ranking and life expectancy, as shown in online supplementary figure $\mathrm{S} 1$, indicates that those countries with better WHO rankings, where the citizens are more likely to have longer life expectancies, also have a higher incidence of this cancer. Another explanation could be the variability in healthcare accessibility among countries with different WHO rankings. Countries with worse rankings are more likely to have poorer healthcare access. Thus, signs and symptoms of pancreatic cancer may be overlooked, thereby causing a decrease in the incidence rate relative to the total cases who die with undiagnosed pancreatic cancer. This will reduce the reported cases both in incidence and in mortality and result in misleading of apparently lower crude rates.

The MIR was calculated with the crude rate ratio of mortality and incidence. In total, 18 countries had MIR values greater than 1.00 , which would not happen in a cohort study. However, the GLOBOCAN database, which provides contemporary estimates of the cancer incidence, mortality and prevalence, is not a cohort study. Other factors, such as control of the denominator time, underestimation of cancer incidence and database quality, might account for this issue. ${ }^{23}$ Therefore, we excluded countries with poor or unknown availability data according to the ranking of GLOBOCAN 2012 to reduce the information and data bias.

Our study has a large sample size, but it still has some limitations. The GLOBOCAN database collected data from various countries to observe cancer epidemiology. The data were also scored according to the quality of data acquisition. We did not include those countries with poor or unknown availability of mortality/incidence data to reduce the bias, which leads to incompleteness of the data collection and reduces the generalisability of the results. Moreover, patients with early disease might not be diagnosed and not be included in this database. We also did not record the risk factors among countries, such as smoking percentage, long-term diabetes mellitus and chronic pancreatitis, and these risk factors may play important roles in determining the incidence and mortality rates among different countries and regions. ${ }^{24-28}$ We also only collected cross-sectional data for 1 year, so the data may not accurately present the actual trend of the disease. Another limitation is the use of WHO ranking: this grading system was established in 2000, so it may not precisely reflect the current situation for healthcare systems among different countries, although the correlations with life expectancy and e/ GDP assure some of its credibility. Despite these limitations, our study shows higher incidence and mortality rates of pancreatic cancer in more developed regions and countries with better WHO rankings. However, the MIRs

(B)

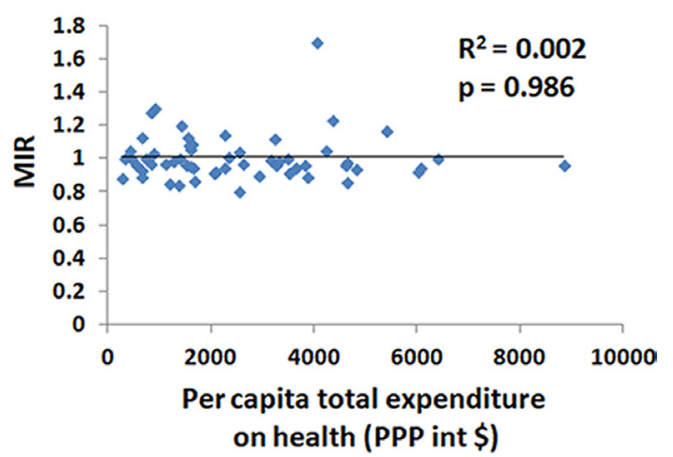

Figure 3 The per capita total expenditure on health in (A) US\$ and (B) purchasing power parity at international dollar rate (PPP) were not associated with the mortality-to-incidence ratio (MIR) of pancreatic cancer. 
of the countries seem to have no association with their WHO rankings and e/GDP.

\section{Author affiliations}

${ }^{1}$ School of Medicine, Chung Shan Medical University, Taichung, Taiwan

${ }^{2}$ Institute of Medicine, Chung Shan Medical University, Taichung, Taiwan

${ }^{3}$ Department of Surgery, Chung Shan Medical University Hospital, Taichung, Taiwan

${ }^{4}$ Department of Urology, National Taiwan University Hospital, Taipei, Taiwan

${ }^{5}$ Department of Rehabilitation, Kaohsiung Veterans General Hospital, Kaohsiung,

Taiwan

${ }^{6}$ Division of Gastroenterology and Hepatology, Department of Internal Medicine,

Chung Shan Medical University Hospital, Taichung, Taiwan

${ }^{7}$ Department of Urology, Chung Shan Medical University Hospital, Taichung, Taiwan

${ }^{8}$ Division of Endocrinology, Department of Internal Medicine, Chung Shan Medical

University Hospital, Taichung, Taiwan

Contributors Conception and design: W-WS; acquisition of data: C-MP, H-LL; analysis and interpretation of data: M-CT, C-CW; drafting of the manuscript: $\mathrm{C}-\mathrm{YH}$, S-YW; critical revision of the manuscript: S-LC, C-CL, C-NH; statistical analysis: C-MP, H-LL, W-WS; supervision: W-WS

Funding This research received no specific grant from any funding agency in the public, commercial or not-for-profit sectors.

Competing interests None declared.

Patient consent Not required.

Provenance and peer review Not commissioned; externally peer reviewed.

Data sharing statement There was no additional unpublished data.

Open access This is an open access article distributed in accordance with the Creative Commons Attribution Non Commercial (CC BY-NC 4.0) license, which permits others to distribute, remix, adapt, build upon this work non-commercially, and license their derivative works on different terms, provided the original work is properly cited and the use is non-commercial. See: http://creativecommons.org/ licenses/by-nc/4.0/

(C) Article author(s) (or their employer(s) unless otherwise stated in the text of the article) 2018. All rights reserved. No commercial use is permitted unless otherwise expressly granted.

\section{REFERENCES}

1. Siegel RL, Miller KD, Jemal A. Cancer Statistics, 2017. CA Cancer J Clin 2017;67:7-30.

2. Siegel RL, Miller KD, Jemal A. Cancer statistics, 2016. CA Cancer J Clin 2016:66:7-30.

3. Kamisawa T, Wood LD, Itoi T, et al. Pancreatic cancer. The Lancet 2016;388:73-85.

4. Malvezzi M, Bertuccio P, Levi F, et al. European cancer mortality predictions for the year 2014. Ann Oncol 2014;25:1650-6.

5. Yip D, Karapetis C, Strickland A, et al. Chemotherapy and radiotherapy for inoperable advanced pancreatic cancer. Cochrane Database Syst Rev 2006;3:CD002093.

6. Higuera O, Ghanem I, Nasimi R, et al. Management of pancreatic cancer in the elderly. World J Gastroenterol 2016;22:764-75.

7. Wang SC, Sung WW, Kao YL, et al. The gender difference and mortality-to-incidence ratio relate to health care disparities in bladder cancer: National estimates from 33 countries. Sci Rep 2017;7:4360.
8. Choi E, Lee S, Nhung BC, et al. Cancer mortality-to-incidence ratio as an indicator of cancer management outcomes in Organization for Economic Cooperation and Development countries. Epidemiol Health 2017;39: e2017006.

9. Sunkara V, Hebert JR. The colorectal cancer mortality-to-incidence ratio as a potential cancer surveillance measure in Asia. Asian Pac $J$ Cancer Prev 2016;17:4323-6.

10. Sunkara V, Hébert JR. The colorectal cancer mortality-to-incidence ratio as an indicator of global cancer screening and care. Cancer 2015;121:1563-9.

11. Asadzadeh Vostakolaei F, Karim-Kos HE, Janssen-Heijnen ML, et al. The validity of the mortality to incidence ratio as a proxy for sitespecific cancer survival. Eur J Public Health 2011;21:573-7.

12. Hébert JR, Daguise VG, Hurley DM, et al. Mapping cancer mortalityto-incidence ratios to illustrate racial and sex disparities in a high-risk population. Cancer 2009;115:2539-52.

13. Cordero-Morales A, Savitzky MJ, Stenning-Persivale K, et al. Conceptual considerations and methodological recommendations for the use of the mortality-to-incidence ratio in time-lagged, ecologicallevel analysis for public health systems-oriented cancer research. Cancer 2016;122:486-7.

14. DaVee T, Coronel E, Papafragkakis C, et al. Pancreatic cancer screening in high-risk individuals with germline genetic mutations. Gastrointest Endosc 2018;87:1443-50.

15. Sidaway P. Pancreatic cancer: New biomarkers improve standard screening. Nat Rev Clin Oncol 2017;14:262.

16. Simon K. Colorectal cancer development and advances in screening. Clin Interv Aging 2016;11:967-76.

17. Suresh T, Chari M, Kelly K. Early detection of sporadic pancreatic cancer, 2015.

18. Herreros-Villanueva M, Bujanda L. Non-invasive biomarkers in pancreatic cancer diagnosis: what we need versus what we have. Ann Transl Med 2016;4:134.

19. Dewitt J, Devereaux BM, Lehman GA, et al. Comparison of endoscopic ultrasound and computed tomography for the preoperative evaluation of pancreatic cancer: a systematic review. Clin Gastroenterol Hepatol 2006;4:717-25. quiz 664.

20. Ansari D, Gustafsson A, Andersson R. Update on the management of pancreatic cancer: surgery is not enough. World $\mathrm{J}$ Gastroenterol 2015;21:3157-65.

21. Chen SL, Wang SC, Ho CJ, et al. Prostate cancer mortality-toincidence ratios are associated with cancer care disparities in 35 countries. Sci Rep 2017;7:40003.

22. Tsai MC, Wang CC, Lee HL, et al. Health disparities are associated with gastric cancer mortality-to-incidence ratios in 57 countries. World J Gastroenterol 2017;23:7881-7.

23. Sunkara V, Hébert JR. The application of the mortality-to-incidence ratio for the evaluation of cancer care disparities globally. Cancer 2016;122:487-8.

24. Yadav D, Lowenfels AB. The epidemiology of pancreatitis and pancreatic cancer. Gastroenterology 2013;144:1252-61.

25. Campa D, Pastore M, Capurso G, et al. Do pancreatic cancer and chronic pancreatitis share the same genetic risk factors? A PANcreatic Disease ReseArch (PANDoRA) consortium investigation. Int J Cancer 2018;142:290-6.

26. Kirkegård J, Mortensen FV, Cronin-Fenton D. Chronic pancreatitis and pancreatic cancer risk: a systematic review and meta-analysis. Am J Gastroenterol 2017;112:1366-72.

27. Li BR, Mao GP, Hu LH, et al. Pancreatic cancer in patients with recently diagnosed chronic pancreatitis. Am J Gastroenterol 2015:110:773-4.

28. Pan J, Xin L, Wang D, et al. Risk factors for diabetes mellitus in chronic pancreatitis: a cohort of 2011 patients. Medicine 2016;95:e3251. 